\title{
$N$-Substituent effects of camphor-derived $\beta$-amino alcohols on the stereoselectivity of the oxazaborolidine-catalyzed borane reduction of acetophenone
}

\author{
Kuangsen Sung,* Gin-Ian Cheng, and Yi-Hui Chen \\ Department of Chemistry, National Cheng Kung University, Tainan, Taiwan \\ E-mail: kssung@mail.ncku.edu.tw
}

\begin{abstract}
Structure-stereoselectivity relationship in modifying chiral ligands usually sheds light on the ligand structure with better stereoselectivity. The camphor-derived $\beta$-amino alcohol 5a with a primary amino group $[\mathrm{R}=\mathrm{H}(84 \%$ ee $)]$ carries out better ee in the oxazaborolidine-catalyzed borane reduction of acetophenone than the ones $\mathbf{5 b} \sim \mathbf{e}$ with secondary amino groups $[\mathrm{R}=n$ - $\mathrm{Bu}$ (11\% ee), $i$-Bu (19\% ee), $i$-Pr (13\% ee), benzyl (9\% ee)].
\end{abstract}

Keywords: Asymmetric synthesis, borane reduction, amino alcohol

\section{Introduction}

Enantioselective reduction of prochiral ketones to form chiral secondary alcohols is one of important issues in organic synthesis. ${ }^{1}$ Oxazaborolidine-catalyzed asymmetric borane reduction of prochiral ketones has been one of important methods to carry out this transformation. ${ }^{2-11}$ Among the oxazaborolidine catalysts, $\mathbf{1}^{3}, \mathbf{2}^{2 \mathrm{a}}, \mathbf{3}^{2 \mathrm{a}}, \mathbf{4}^{2 \mathrm{a}}$, and $\mathbf{5}^{4}$ successfully reduced acetophenone in $95 \%, 97 \%, 86 \%, 94 \%$, and $79 \%$ ee, respectively. It was found that bulky substituents on the carbinol carbon of these $\beta$-amino alcohols performed better enantioselective reduction of acetophenone $^{2 \mathrm{a}}$ and $\mathbf{2}$ with a secondary amine of $\beta$-amino alcohol is one of the most efficient chiral catalysts in the reduction of acetophenone $\left(97 \%\right.$ ee). ${ }^{2 \mathrm{a}}$

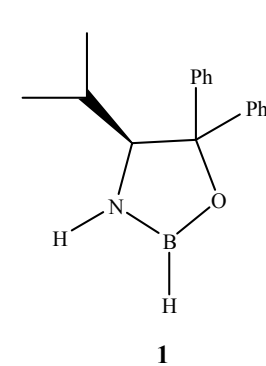

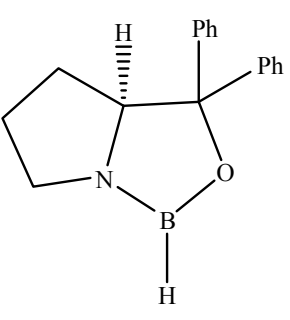

2

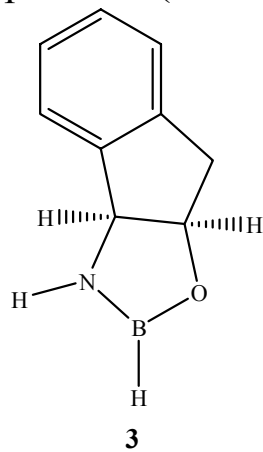

3

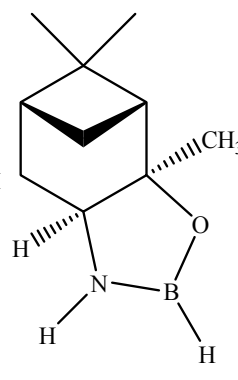

4

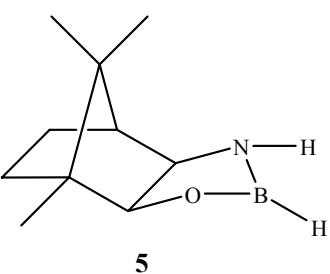


A chiral ligand provides chiral environment in the catalytic asymmetric synthesis, so studies concerning the relationship between the ligand structure and asymmetric induction can be used to develop more efficient catalysts. In this article, we use acetophenone as a model substrate of prochiral ketones and study $N$-substituent effects of camphor-derived $\beta$-amino alcohols on the stereoselectivity of the oxazaborolidine- catalyzed borane reduction.

\section{Results and Discussion}

The oxazaborolidine-catalyzed borane reduction of acetophenone was carried out according to the known procedure, ${ }^{4,5}$ whereas, $10 \%$ of oxazaborolidine catalyst was prepared in situ by mixing 1 equivalent of camphor-derived $\beta$-amino alcohol $(\mathbf{5 a}, \mathbf{5 b}, \mathbf{5 c}, \mathbf{5 d}$, or $\mathbf{5 e})$ with 8.5 equivalent of $\mathrm{BH}_{3} \cdot \mathrm{Me}_{2} \mathrm{~S}$ at room temperature for one hour. The following oxazaborolidinecatalyzed borane reduction of acetophenone was carried out at room temperature for 3 hours. By following this method, the camphor-derived $\beta$-amino alcohol 5a, which is a primary amine, led the reduction of acetophenone to $(S)-6$ with $84 \%$ ee, which is consistent with the literature result of $79 \%$ ee. ${ }^{4}$ When the camphor-derived $\beta$-amino alcohol $(\mathbf{5 b}, \mathbf{5 c}, \mathbf{5 d}$, or $\mathbf{5 e})$ with a secondary amine group was applied to the oxazaborolidine-catalyzed borane reduction of acetophenone, ee values of the product $(S)-6$ dramatically decrease in spite of high yield. (Table 1)

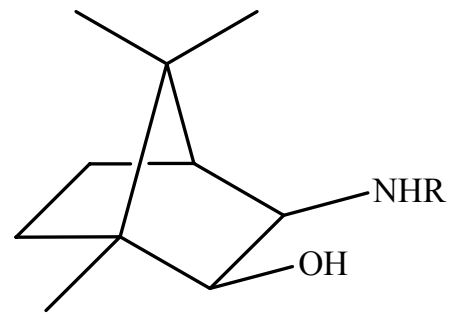

5a: $\mathrm{R}=\mathrm{H}$

$\mathbf{5 b}: \mathrm{R}=n-\mathrm{Bu}$

5c $: \mathrm{R}=i-\mathrm{Bu}$

5d : $\mathrm{R}=i-\mathrm{Pr}$

5e : $\mathrm{R}=$ Benzyl 
Table 1. Oxazaborolidine-catalyzed borane reduction of acetophenone
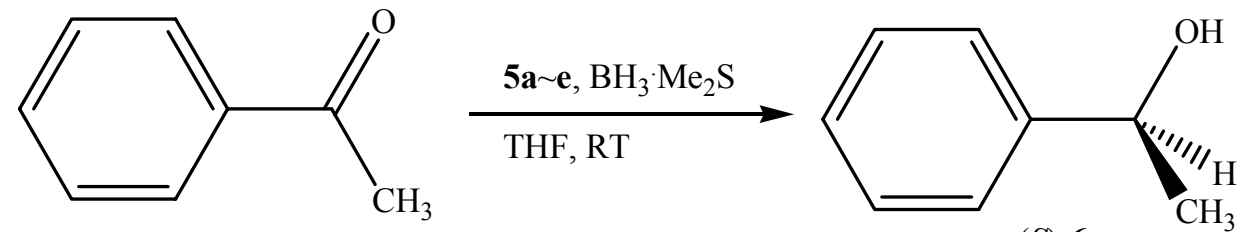

(S)-6

\begin{tabular}{cccc}
\hline Chiral ligand & $\mathrm{R}$ on chiral ligand & Yield (\%) & ee (\%) ${ }^{\mathrm{a}}$ of product \\
\hline $\mathbf{5 a}$ & $\mathrm{H}$ & 97 & 84 \\
$\mathbf{5 b}$ & $n-\mathrm{Bu}$ & 90 & 11 \\
$\mathbf{5 c}$ & $i-\mathrm{Bu}$ & 96 & 19 \\
$\mathbf{5 d}$ & $i-\mathrm{Pr}$ & 97 & 13 \\
$\mathbf{5 e}$ & $\mathrm{Benzyl}$ & 86 & 9 \\
\hline
\end{tabular}

a. The major product is $(S)$-6. The ee was determined by HPLC analysis with a Chiralcel OD-RH column and the configuration was assigned based on the result of the $\mathrm{BH}_{3} \cdot \mathrm{Me}_{2} \mathrm{~S} / \mathbf{5 a}$-catalyzed borane reduction of acetophenone, HPLC analysis, and the literature. ${ }^{4}$

According to the Corey proposed mechanism, ${ }^{2 a}$ the oxazaborolidine-catalyzed borane reduction involving camphor-derived $\beta$-amino alcohol (5a $\sim \mathbf{e})$ was expected to go through TS1, TS2, TS3, or TS4, where the first equivalent of $\mathrm{BH}_{3}$ is consumed to produce the oxazaborolidine catalyst after reaction with the corresponding amino alcohol. This complex serves as a Lewis acid to activate acetophenone. The second equivalent of $\mathrm{BH}_{3}$ works as a hydride donor to reduce acetophenone. The TS1 and TS2 have oxazaborolidine ring cis fusion with formation of sixmember ring, while trans fusion was found in the TS3 and TS4. Both TS2 and TS4 suffer from steric repulsion between B-H and methyl group on the camphor skeleton. However, cis fusion has less angle strain than trans fusion and thus, TS1 is more stable than TS3 and the product $(S)$ 6, which results from TS1, is expected to be the major. That is consistent with the experimental results.

The presence of large $\mathrm{R}$ group in the camphor-derived $\beta$-amino alcohol results in more steric repulsion between the R group and the camphor skeleton in TS1 and TS3. In addition, TS3 and TS4 suffer an additional trans-fusion angle strain and TS4 is further destabilized by steric repulsion between B-H and methyl group of the camphor skeleton. Thus, TS1 is expected to be the most stable among TS1, TS3, and TS4. Even though there is very little steric repulsion between the R group and the camphor skeleton in TS2, there is also a steric repulsion between B$\mathrm{H}$ and methyl group on the camphor skeleton. According to the experimental results, when $\mathrm{R}$ group in the camphor-derived $\beta$-amino alcohol is larger, it is likely that the oxazaborolidinecatalyzed borane reduction follows the route via TS1 only a little bit faster than other routes via TS2, TS3, and TS4 because of increasing repulsive destabilization between the R group and the camphor skeleton in TS1, resulting in decrease of ee values. 
Finally, based on our experiments and the above discussion we conclude that $N$-alkyl substituents in the camphor-derived $\beta$-amino alcohols decrease stereoselectivity of the oxazaborolidine-catalyzed borane reduction of acetophenone.
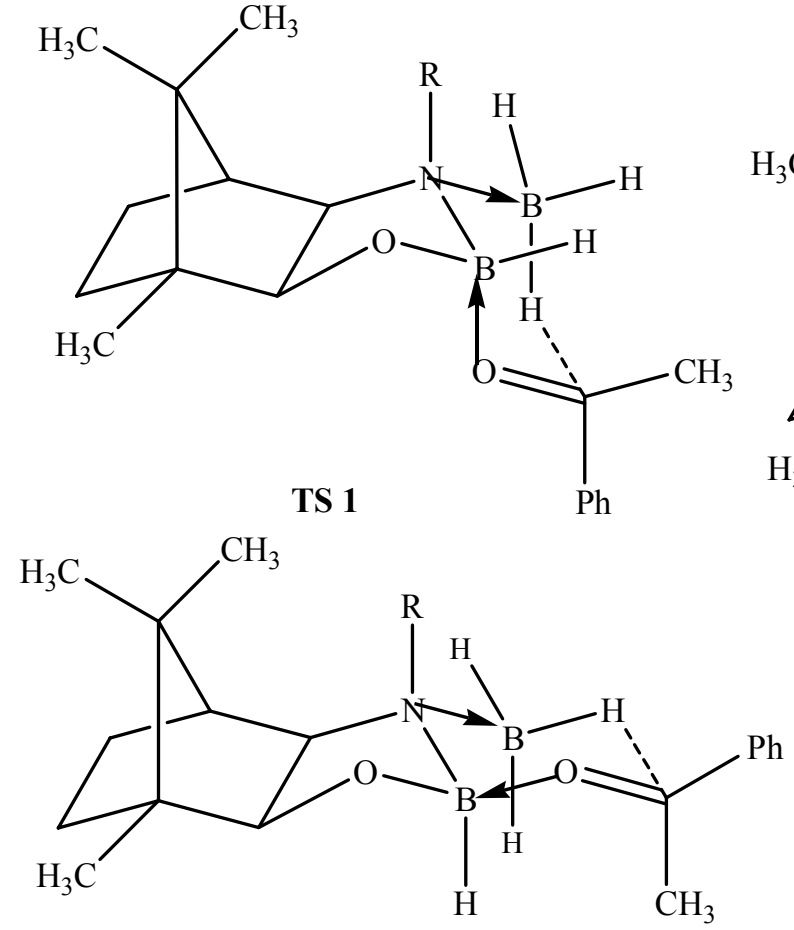

TS 3

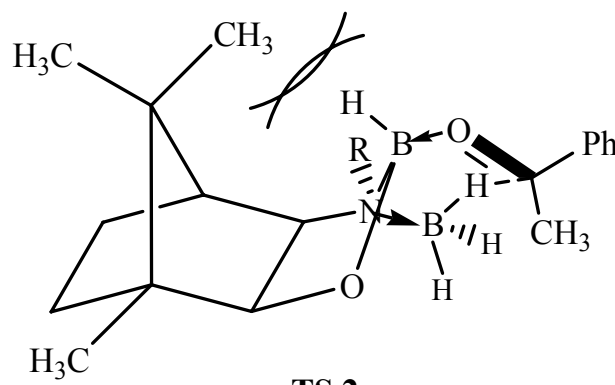

TS 2

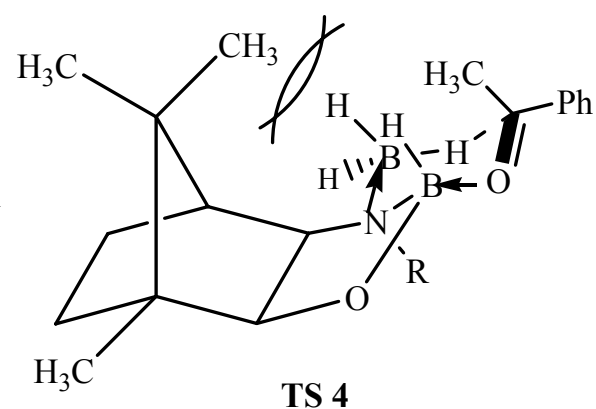

\section{Experimental Section}

General Procedures. Reagents were used as they were obtained from commercial sources. Chiral $\beta$-amino alcohols $\mathbf{5 a} \sim \mathbf{e}$ were prepared according to the literature. ${ }^{12}$

\section{General procedure for the oxazaborolidine-catalyzed borane reduction of acetophenone}

The camphor-derived $\beta$-amino alcohol $5(0.2 \mathrm{mmol})$ was dissolved in $3 \mathrm{~mL}$ of dry THF in a twonecked flask fitted with a septum in a nitrogen atmosphere. The $0.3 \mathrm{mmol}$ of $2 \mathrm{M} \mathrm{BH}_{3} \cdot \mathrm{Me}_{2} \mathrm{~S}$ in THF was added to this solution and the mixture was stirred for one hour at room temperature. Then another $1.4 \mathrm{mmol}$ of $2 \mathrm{M} \mathrm{BH} \cdot \mathrm{Me}_{2} \mathrm{~S}$ in THF was added to this solution and the reaction mixture was stirred for another 10 minutes. Acetophenone $(0.240 \mathrm{~g}, 2 \mathrm{mmol})$ in $4 \mathrm{~mL}$ THF was added dropwise to this solution. After 3 hours of reaction, the excess of borane was destroyed by dropwise addition of cold $0.1 \mathrm{M} \mathrm{HCl}(\mathrm{aq})$. The reaction mixture was extracted with ether. The organic layer was dried over anhydrous $\mathrm{Na}_{2} \mathrm{SO}_{4}$ and concentrated. The crude product was purified by column chromatography with silica gel using a mixture of hexane and ethyl acetate as the eluent to get alcohol $(S)$-6. The degree of conversion was determined by proton NMR 
spectroscopy. The enantiomeric excesses was determined by HPLC analysis with a Chiralcel OD-RH column, mobile phase: $40 \%$ acetonitrile in $\mathrm{H}_{2} \mathrm{O}$, UV detector: $257 \mathrm{~nm}$, and flow rate: 0.3 $\mathrm{ml} / \mathrm{min}$. The retention time for $(R)-6$ and $(S)-6$ are 11.67 and $12.55 \mathrm{~min}$, respectively.

\section{Acknowledgements}

Financial support from the National Science Council of Taiwan (NSC 95-2113-M-006-008) is gratefully acknowledged. We thank Mr. Chen for some synthetic work.

\section{Reference}

1. (a) Noyori, R. Asymmetric Catalysis in Organic Synthesis; John Wiley: New York, 1994. (b) Catalytic Asymmetric Synthesis; I. Ojima, Ed.; VCH: Berlin, 1993.

2. (a) Corey, E. J.; Helal, C. J. Angew. Chem., Int. Ed. Engl. 1998, 37, 1986. (b) Corey, E. J.; Bakshi, R. K.; Shibata, S. J. Am. Chem. Soc. 1987, 109, 5551. (c) Corey, E. J.; Bakshi, R. K.; Shibata, S.; Chen, C.; Singh, V. K. J. Am. Chem. Soc. 1987, 109, 7925.

3. Itsuno, S.; Ito, K.; Hiraou, A.; Nakahama, S. J. Chem. Soc., Chem. Commun., 1983, 469.

4. Rao, M.; Santhi, V. Tetrahedron: Asymmetry 2000, 11, 3553.

5. Puigjaner, C.; Vidal-Ferran, A.; Moyano, A.; Pericas, M. A.; Riera, A. J. Org. Chem. 1999, 64, 7902.

6. Pinho, P.; Guijarro, D.; Andersson, P.G. Tetrahedron 1998, 54, 7897.

7. Wallbaum, S.; Martens, J. Tetrahedron: Asymmetry 1992, 3, 1475.

8. Simone, B.; Savoia, D. D.; Tagliavini, Umani-Ronchi, E. A. Tetrahedron: Asymmetry 1995, 6,301 .

9. Giffels, G.; Beliczey, J.; Felder, M.; Kragl, U. Tetrahedron: Asymmetry 1998, 9, 691.

10. Jones, S.; Atherton, J. C. C. Tetrahedron: Asymmetry 2000, 11, 4543.

11. Bolm, C.; Derrien, N. ; Seger, A. Chem. Commun. 1999, 2087.

12. Cheng, G. -I.; Shei, C. -T.; Sung, K. Chirality 2007, 19, 235. 